\title{
Dedication to late Dale Han, MD
}

\author{
Giorgos C. Karakousis ${ }^{1}$ · Stanley P. Leong ${ }^{2}$. Jonathan S. Zager ${ }^{3,4}$
}

Accepted: 7 February 2022 / Published online: 7 March 2022

(c) The Author(s), under exclusive licence to Springer Nature B.V. 2022

Dr. Dale Han was born on 16 June 1976 in New York and passed away unexpectedly on October 2, 2021 in Bethany, Oregon. He received his undergraduate and medical school degrees at the State University of New York at Stony Brook. He went on to complete his general surgery residency training at the Hospital of the University of Pennsylvania, prior to going on to complete his Complex General Surgical Oncology fellowship at the H Moffitt Cancer Center in Tampa, Florida. Dr. Han was one of the first surgical oncology fellows in the country to be board-certified in Complex General Surgical Oncology after fellowship and then took a faculty position at Yale University, where he quickly gained national recognition in the field of melanoma through multiple publications and presentations. In 2018, he was recruited to Oregon Health \& Science University as an Associate Professor, where he continued to take care of multitudes of grateful patients with melanoma and other cutaneous and soft tissue malignancies, and continued his research in melanoma, with a specific interest in prognostic biomarkers. He remained very active in the Society of Surgical Oncology and was a member of the Melanoma Disease Site Working Group. In addition, Dr. Han served as the Chair of the Scientific

To be published in an upcoming Special Issue of Clinical and Experimental Metastasis: Novel Frontiers in Cancer Metastasis.

Jonathan S. Zager

Jonathan.Zager@moffitt.org

Giorgos C. Karakousis

Giorgos.karakousis@pennmedicine.upenn.edu

Stanley P. Leong

Stanley.Leong@ucsf.edu

1 Hospital of the University of Pennsylvania, Philadelphia, PA, USA

2 University of California San Francisco School of Medicine, San Francisco, CA, USA

3 Department of Cutaneous Oncology, Moffitt Cancer Center, Tampa, FL, USA

4 Department of Oncologic Sciences, University of South Florida, Morsani College of Medicine, Tampa, FL, USA
Committee of the Sentinel Lymph Node Working Group. In this Special Issue: Novel Frontiers of Cancer Metastasis, Dr. Han was the first author of the review article on: Current management of melanoma patients with nodal metastases.

Dr. Dale Han was a kind, compassionate surgeon, researcher and teacher who deeply cared about his patients and was devoted to his family. He was passionate about outcomes-based research and was involved in numerous publications that helped shape the clinical care of melanoma and non-melanoma skin cancer patients during his short, but incredibly productive, academic career. His dedication to improving patient outcomes was infectious and he inspired all of those around him, including colleagues, trainees and friends. Dale's legacy will prevail; he will forever leave his mark in the care of patients with melanoma, and memories of Dale's smiling face in meetings and passion for knowledge will continue to inspire us to try to reach a better understanding of diseases as we strive to improve the lives of our patients. We are forever grateful for having known Dale. We will miss him dearly, our colleague and our friend. We dedicate this Special Issue to Dr. Dale Han.

Publisher's Note Springer Nature remains neutral with regard to jurisdictional claims in published maps and institutional affiliations. 